\title{
Magneto-piezoresistivity in iron particle-filled silicone: An alternative outlook for reading magnetic field intensity and direction
}

\author{
V. Iannotti ${ }^{1}$, G. Ausanio ${ }^{1}$, L. Lanotte ${ }^{2}$, L. Lanotte L $^{*}$ \\ ${ }^{1}$ CNR-SPIN and Department of Physics, University of Naples 'Federico II', Piazzale V. Tecchio 80, I-80125 Napoli, Italy \\ ${ }^{2}$ Laboratoire Charles Coulomb, Université de Montpellier, Place Eugène Bataillon - Centre de Biochimie Structurale, \\ 29 rue de Navacelles, 34095 Montpellier, France
}

Received 17 June 2015; accepted in revised form 26 August 2015

\begin{abstract}
Elastomagnetic effect (strain induced by magnetic field application) and piezoresistivity (change of electron conductivity due to an induced strain) are coupled in composite materials constituted by magnetic and conductive microparticles into an elastic matrix. On the basis of these effects, the principle of a new method to read magnetization direction changes, in a random sequence, is proposed and experimentally demonstrated. We have produced new composite magnetopiezoresistive samples, constituted of thin chip shaped Fe microparticles inside a silicone matrix, which under an applied magnetic field along their longitudinal axis, undergo an induced strain depending on the local magnetization direction. The resulting resistivity change can be easily detected and used to deduce the local magnetization direction. The magnetization and strain processes are reversible so that after the removal of external magnetizing field the sample is ready for new measurements. A demonstrator prototype has been conceived, produced and tested. The experimental results provide interesting data encouraging to continue the research towards nano-scale devices in order to pursue the intriguing perspective to achieve a magnetic field gradient sensitivity able to reveal magnetization of semipermanent nanomagnets, polarized 'up' and 'down'.
\end{abstract}

Keywords: smart polymers, magneto-piezoresistivity, material testing, magnetic direction sensors

\section{Introduction}

Composite materials based on polymer matrix filled by conductive or magnetic micro/nano particles have shown very interesting mechanical and electrical properties [1-3], and are very promising for application in aeronautical, mechanical and civil engineering [4-7]. In particular, several investigations have highlighted the existence of a giant piezoresistive effect (the electron conductivity can change by 9 orders of magnitude) in composite materials constituted by a silicone matrix filled by conductive microparticles at a volume fraction around percolation threshold [8-10].
On the other hand, composites made of magnetic particles homogeneously dispersed into an elastic matrix (generally known as magnetorheologic materials) present improved mechanical, thermal and magneto-electric properties under the application of an external magnetic field, during the production or onto the produced composite [11-14]. In particular, the inverse elastomagnetic effect is exhibited: strain appears when its state of magnetization is changed, due to the alignment or rotation of magnetic particles and it is not depending on the intrinsic magnetostriction of the particles [15-19].

\footnotetext{
${ }^{*}$ Corresponding author, e-mail: lanotte@na.infn.it

(C) BME-PT
} 
When the filling particles inside the polymer matrix are magnetic and conductive ( $\mathrm{Fe}, \mathrm{Ni}, \mathrm{FeSi}$, etc.) concomitant effects of piezoresistivity and elastomagnetism appear in the same sample, and their optimization is obtained by a proper combination of the particle size, shape, volume fraction, magnetic permeability and electrical conductivity. In this way, if the filling particles have no preferential orientation, the application of a longitudinal gradient of magnetic induction produces a sample sensible elongation, independent of both intrinsic magnetostriction and particle rotations. Indeed, this strain is prominently produced by the forces applied by the magnetic field gradient (elastomagnetism). As a result of this strain, a decrease of average distance among the particles and a consequent decrease in resistivity occur (piezoresistivity). These are the fundamental mechanisms in the coupling of elastomagnetism and piezoresistivity which produce the magneto-piezoresistive (MPR) effect extensively treated in [20] and [21]. A magneto-piezoresistive sensitivity (change of resistivity on the inducing magnetic field gradient) higher than $10^{11} \Omega \mathrm{m} / \mathrm{T}$ has been both predicted by theoretical models and experimentally detected $[20,21]$.

The main objective of this paper is to establish, by means of some basic experiments in the millimeter scale range, that the MPR effect can be used to detect the change of magnetic polarization direction at a magnetization intensity similar to that produced by the semi-permanent nanomagnets of a magnetic memory.

It does not seem easy to produce samples MPR through the nanowires by keeping the used magnetic interaction between nano-particles of iron in it, but there are some encouraging data in the literature $[22,23]$. Nevertheless, the methodology, and operation principles of the sensor proposed here, are very promising and intriguing, and can constitute a basic step to open new perspectives in the field of magnetic reading heads with competitive performance and costs in comparison with standard GMR spin valve.

\section{Experimental}

\subsection{Sample preparation}

Iron micro-particles have been used as magnetic filling charge of the MPR composite samples used for this investigation. They have been obtained by mechanical crushing of a pure Fe foil (pureness

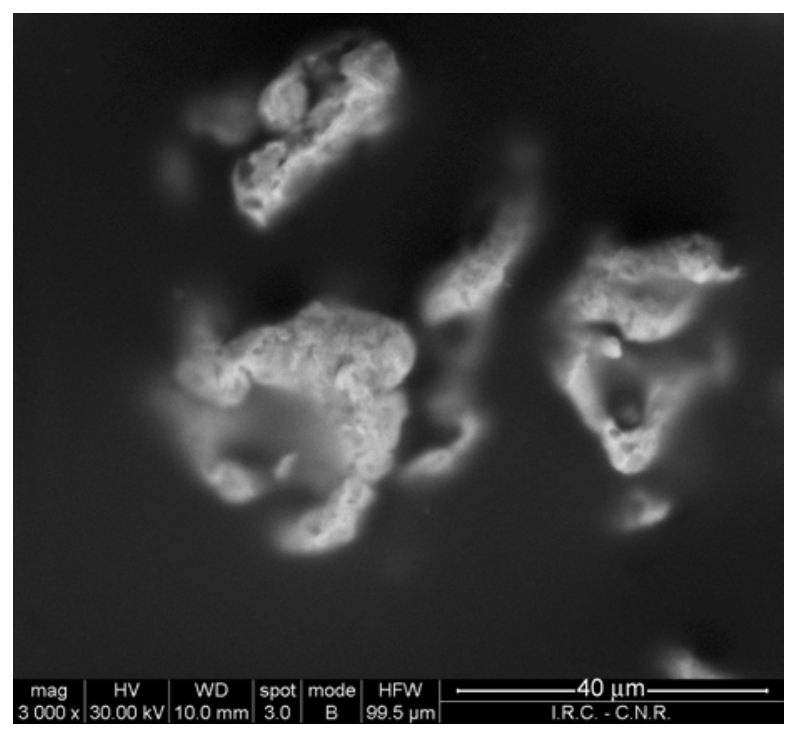

Figure 1. SEM image of iron particles used as filling magnetic/conductive charge

99,99, cod.338141,Aldrich Chemical Company, Inc., U.S.A.) at room temperature and they exhibit a peculiar irregular shape similar to a shrunken little plate or a chip (Figure 1). The elaboration of several SEM images evidenced an average thickness of $7 \mu \mathrm{m}$ and an average size of particles major axis around $37 \mu \mathrm{m}$.

Magnetic particles, at the volume fraction of $39 \%$, were mechanically dispersed into liquid silicone (Essil 291, Axon, France), and hand spatulated carefully for 1 hour, in order to obtain both a homogeneous particle distribution and a good intrusion of silicone among the particle protrusions. After 1 hour of degassing process, at a constant temperature of $40^{\circ} \mathrm{C}$, the solidification process of the polymeric matrix was triggered by adding, and mixing for 20 minutes, a reticulating agent at a percentage of 12\% (Essil 292 Catalyseur, Axon, France). Immediately after mixing with the crosslinking agent, the mixture was slowly injected into a Teflon mould $2 \times 2 \times 25 \mathrm{~mm}^{3}$. The little bar of composite (polymer matrix + magnetic particles) was peeled off from the mould after 48 hours. The sample length can be easily reduced by a laboratory knife. All the process has been described in some detail in previous papers [9, 20,21]. No methodology has been applied to induce a preferential orientation of the Fe microparticles; therefore they resulted homogeneously distributed and randomly oriented. The peculiar morphology of magnetic particles used in this work is different from the one employed in previous investigations (pseudospherical morphology with moderate roughness). 
The reason of this choice is to improve composite material performances, delineated on the basis of previous studies [9, 20,21], in order to achieve three specific characteristics: i) bigger size particles; ii) better silicone entrapping among particle cavities during the mechanical dispersion process to isolate each particle from nearest ones; iii) lower transversal contraction able to give particle contact as consequence of the irregular surface protrusions; iv) preferential in plane magnetization of a single particle due to shape anisotropy. The positive effects of these listed material characteristics were verified by means of the sample performance as will be shown and discussed in Section 3.

\subsection{Principles of the experimental demonstrator}

The experimental apparatus, specifically arranged to demonstrate the potential of the sensors based on the innovate elastomagnetic composite produced for the present investigation, is shown in Figure 2. The magnet $M$ has a permanent magnetization intensity of $16 \cdot 10^{4} \mathrm{~A} / \mathrm{m}$. It produces an initial polariza-

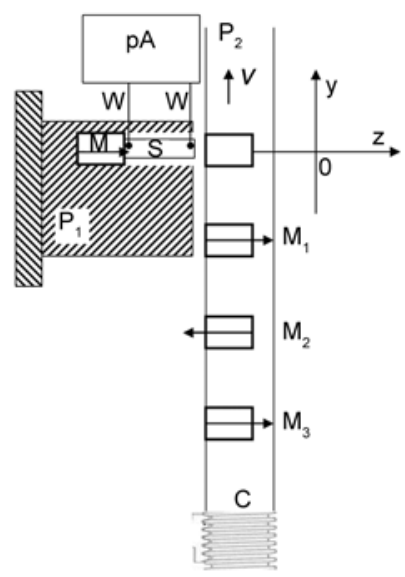

Figure 2. Scheme of the experimental arrangement used to evaluate the capability of a MPR sensor to detect local magnetization direction. S - MPR sample $(2 \mathrm{~mm} \times 2 \mathrm{~mm} \times 6 \mathrm{~mm}) ; \mathrm{M}-$ permanent magnet $(2 \mathrm{~mm} \times 2 \mathrm{~mm} \times 3 \mathrm{~mm})$ producing a static polarization of the MPR sample; $\mathrm{P}_{1}-$ Plexiglas matrix in which $\mathrm{M}$ is fixed and $\mathrm{S}$ is free to have a variable strain under the action of an additional external magnetizing field; $\mathrm{W}-$ microwires for conductive contacts; $\mathrm{pA}$ - HP picoammeter to measuring sample electrical resistance; $\mathrm{P}_{2}$ - sliding Plexiglas holder in which external magnets $\left(\mathrm{M}_{\mathrm{i}}\right.$, similar to $\left.\mathrm{M}\right)$ are embedded; $M_{i=1,2,3 \ldots}$ - permanent magnets having magnetization direction concordant or opposite to $\mathrm{M}$ static polarization; $\mathrm{C}$ - mechanical cursor enabling $\mathrm{P}_{2}$ displacement along $y$ axis, orthogonal to sample longitudinal axis $(z)$. tion status of the MPR material; in this status the sample resistance is $R_{0}$. The magnets $M_{\mathrm{i}}$ (identical in size and magnetization intensity to $M$ ) are embedded in the Plexiglas holder $\mathrm{P}_{2}$ that can run perpendicularly to the longitudinal axis of the MPR sensor core. When a running magnet $M_{\mathrm{i}}$ becomes coaxial with the sample, its magnetizing field adds to initial static polarization induced by the fixed magnet $M$. As we shall see in the following section, the sample length was designed so that the fields of $M$ and $M_{\mathrm{i}}$ affect only a half of the sample, the one on the side of $M$ and $M_{\mathrm{i}}$, respectively. On the basis of this experimental expedient, a different MPR core elongation is obtained if the $M_{\mathrm{i}}$ magnetic moment is directed in concordant or opposite direction with respect to $M$ magnetization. According to the MPR model [20, 21], a different sample elongation determines a different decrease of sample initial resistance $R_{0}$. We will use the symbol $R_{\text {up }}$ for the sample resistance when $M$ and $M_{\mathrm{i}}$ magnetization directions are identical, while we will use $R_{\text {down }}$ in case they are opposite.

\section{Results and discussion}

In Figure 3 the longitudinal magnetization cycle of a MPR sample $\left(2 \times 2 \times 6 \mathrm{~mm}^{3}\right)$, produced as described in Section 2, is reported. The magnetization behaviour appears strictly related to both the peculiar morphology of the particles, with a strong shape anisotropy, and the silicone matrix that enables only little rotation of the particles towards the longitudinal magnetizing field axis.

In agreement with these peculiar characteristics of the innovative composite polymer produced for this

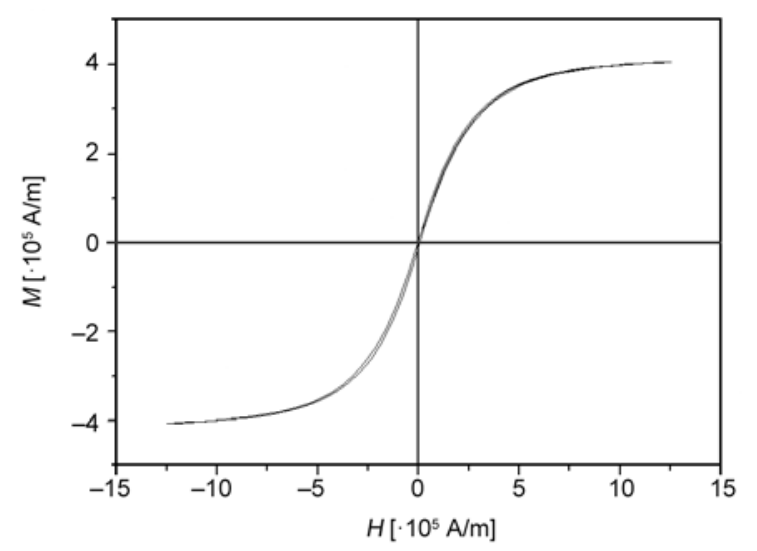

Figure 3. Longitudinal magnetization cycle of the sample constituted by iron microparticles in the volume percentage of $39 \%$ homogeneously dispersed into a silicone matrix. Coercive field is $3900 \mathrm{~A} / \mathrm{m}$ and saturation field higher than $1 \mathrm{MA} / \mathrm{m}$. 
investigation, one observes low values of both coercive field and remnant magnetization, together with a progressive approach to saturation of the magnetization that, above $2 \cdot 10^{5} \mathrm{~A} / \mathrm{m}$, mainly increases for rotation of magnetic moments towards the applied field axis.

When the magnet $M$ is in contact with the MPR sample $\mathrm{S}$ (see scheme in Figure 2) its magnetizing field is not able to homogeneously magnetize all the composite material. In order to monitor this effect, an Hall probe was placed on the MPR sample end located on the opposite side of $M$ magnet (see inset in Figure 4) and, by using samples with variable length 1 , the magnetization induction $B$ obtained in the MPR material versus the distance 1 from the magnet pole was measured. Subsequently, the measure of magnetization induction $B_{0}$ without MPR sample was performed. The graph in Figure 4 was obtained by calculating $M=\left(B-B_{0}\right) / \mu_{0}$ : at a distance of about $3 \mathrm{~mm}$ the magnetization is practically zero. Moreover, also the gradient of magnetic inductions $B$ and $B_{0}$ are negligible at a distance from $M$ higher than $1 / 2$. This means that, when a magnet $M_{\mathrm{i}}$ is put on the opposite side of $M$, one can predict the overlap of the magnetization intensity as reported in Figure 5. Since the sample has a length of $6 \mathrm{~mm}$, practically the magnet $M$ gives polarization of Fe particles in one half of the sample, while magnet Mi influences the opposite half of the sample. Since the magnetic induction gradient and the magnetization intensities are practically zero in the sample center, in any case the half of the composite near $M$ is always magnetized in the same way and undertakes the same attractive force towards $M$, whose longitudinal component is: $F_{1 \mathrm{z}}=$ $\int_{0}^{1 / 2} M_{\mathrm{z}}\left(\partial B_{0 \mathrm{z}} / \partial z\right) \mathrm{d} z$. This force gives an initial con-

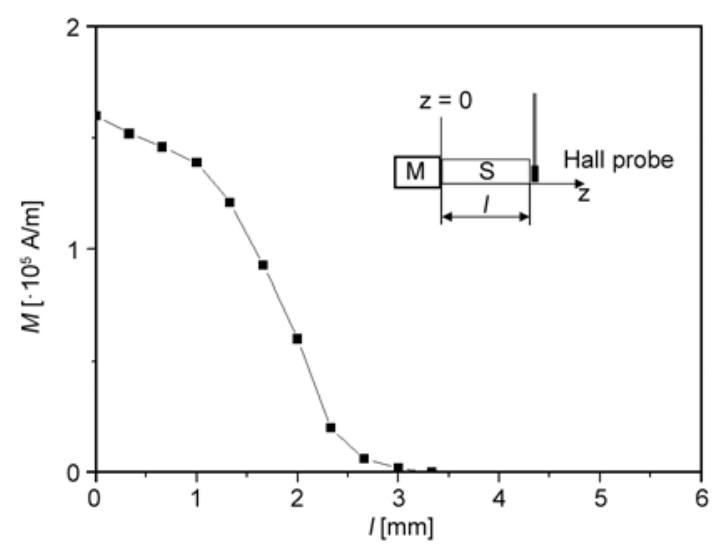

Figure 4. Intensity of magnetization produced statically inside the MPR sample as a function of the distance $l$ from the magnet $M$ traction of the interested sample region which determines the conduction status of the MPR sample when $M_{\mathrm{i}}$ action is absent. When the cursor runs and a $M_{\mathrm{i}}$ magnet aligns with sample axis (Figure 2), its presence does not modify the effect of the fixed magnet $M . M_{\mathrm{i}}$ produces induction field gradient and magnetization only in half the sample, the one closer to $M_{\mathrm{i}}$ itself, and a consequent attractive force with a component: $F_{2 \mathrm{z}}=\int_{1 / 2}^{1} M_{\mathrm{z}}\left(\partial B_{0 \mathrm{z}} / \partial z\right) \mathrm{d} z$. Since the change of longitudinal magnetizing field, applied by $M_{\mathrm{i}}$, produces a sign change of both magnetization and magnetic induction gradient, the last force does not change in intensity and direction with direction change of $M_{\mathrm{i}}$ magnetic moment ('up' or 'down'). In conclusion, the MPR core is attracted by the same force towards $M_{\mathrm{i}}$ magnet in any case ('up' or 'down' configuration). The last attractive force produces the same elongation of the MPR sample and an equal resistance decrease.

On the other side, since magnetization cycles exhibit a practically zero remnant magnetization (Figure 3 ), one can deduce that, also if particle rotations in the elastic matrix occur during the magnetization process, whenever a magnetizing field zero is restored, particle orientations are always randomly distributed around the longitudinal axis of magnetization. This assures that the eventual particle rotations, during the magnetization process in the half sample influenced by $M_{\mathrm{i}}$ gives always a sample elongation independent on magnetization direction. Consequently, also the resistance decrease due to particle rotations is independent of $M_{\mathrm{i}}$ moment direction.

In conclusion, what discriminates the condition 'up' from that 'down' is the fact that the total sensor core strain is also determined by the internal attractive forces that the polarized particles in half sample apply to those ones on the other side. If $M$ and $M_{\mathrm{i}}$ produces the same magnetization directions (configuration 'up'; Figure 5a; see the insert too), the iron particles polarization is the same in all the sample: internal attractive forces are produced that oppose the fixed tensile stress applied by the external magnetic induction gradient and promote a decrement of elongation. On the contrary, if magnetization directions of $M$ and $M_{\mathrm{i}}$ are opposite (configuration 'down'; Figure 5b), iron micro-magnets inside the sample half in proximity of $M$ have a longitudinal magnetic moment anti-parallel with respect those in the sample half closer $M_{\mathrm{i}}$ : repulsive internal forces are present that promote an increment of elongation. Thus, 

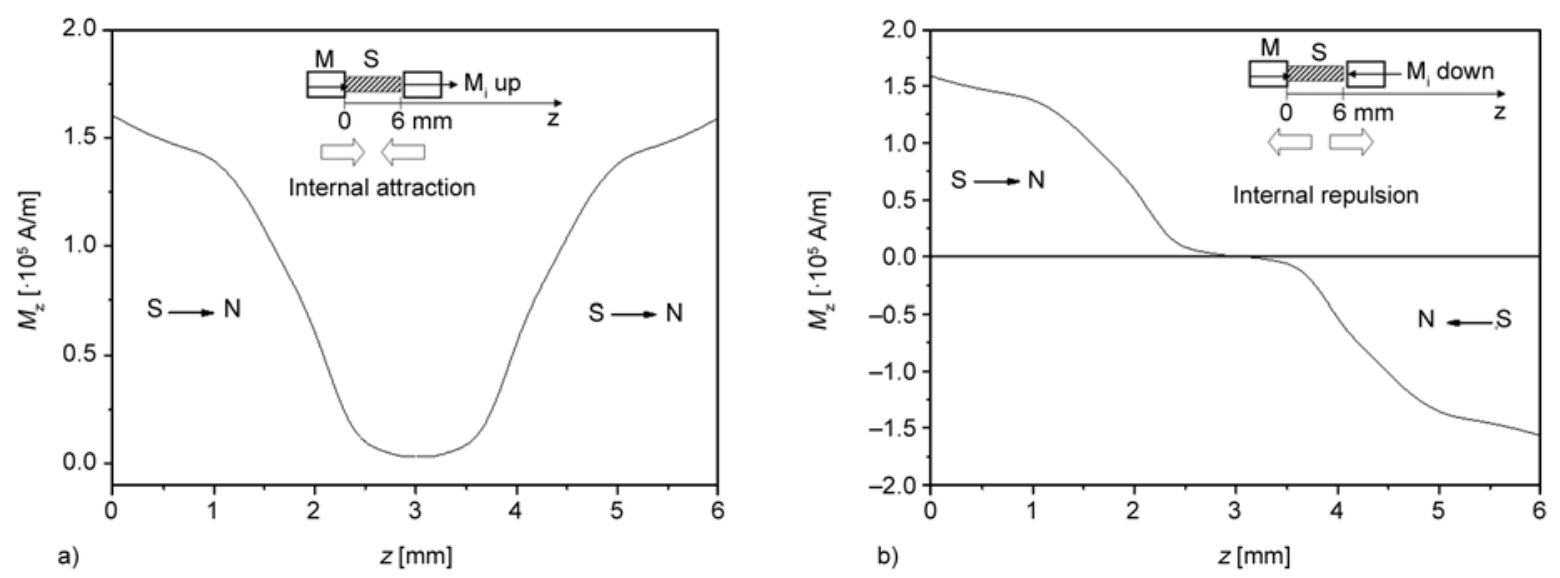

Figure 5. Total behavior of magnetization longitudinal component along the MPR sample as function of the longitudinal coordinate $\mathrm{z}$, in the case of concordant magnetization direction of the external aligned magnets $M$ and $M_{\mathrm{i}}$ (a) and in the case of opposite magnetization directions (b)

in the last case $\left(M_{\text {down }}\right)$ the sample should have a higher elongation and, on turn, a higher decrease of resistance in comparison with the decrement produced in the condition 'up'.

The scheme in Figure 5 is very effective in explaining the experimental evidences as we will describe below.

The MPR sample resistance has been detected by means of the experimental apparatus described in Section 2, at a displacement velocity of the cursor $C+P_{2}$ fixed at $1 \mathrm{~mm} / 10 \mathrm{~s}$.

In Figure 6, $R$ behaviour versus the displacement along $y$ axis is reported, starting from the position shown in Figure 2, namely when the $P_{2}$ hole in front of the MPR sample is an empty one. On the same figure, along the $y$ axis, the different $M_{\mathrm{i}}$ magnets position is shown, in order to deduce the relative position of external magnets with respect to the sensor core longitudinal axis and their different magnetic polarization directions.

The experimental points are centered in the average $R$ value obtained by several measurement runs. The error bars are contained into the graph point size.

The effects of $M_{\text {up }}$ or $M_{\text {down }}$ magnets on sample resistance, qualitatively predicted above, are completely verified.

In particular, the resistance decrease $\left(R_{0}-R_{\text {up }}\right)$, produced by the alignment of $M_{\text {up }}$ magnets with sensor core axis, is around $450 \mathrm{k} \Omega$; while, $M_{\text {down }}$ magnets alignment gives $\left(R_{0}-R_{\text {down }}\right)$ higher than $700 \mathrm{k} \Omega . R_{\text {up }}$ and $R_{\text {down }}$ values have an average difference higher than $250 \mathrm{k} \Omega$. This enable an easy detection of the $M_{\text {up }}$ or $M_{\text {down }}$ alignment and a clear discrimination

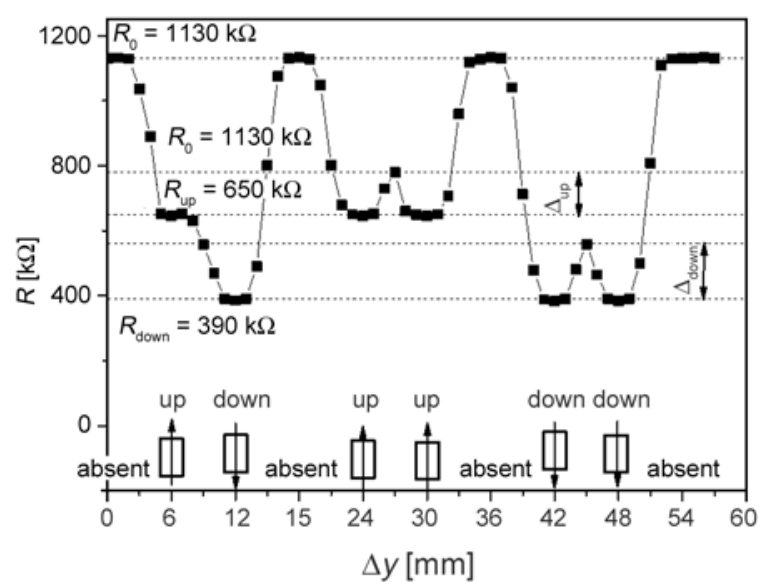

Figure 6. Resistance $R$ of the MPR core measured versus the displacement $\Delta y$ of the $M_{\mathrm{i}}$ magnets, that, sliding along the $y$ axis (Figure 2), align with the sample $z$ axis, in the sequence reported on the bottom of the figure

between the two cases. Moreover, also the signals produced by a sequence of equal polarization directions, $M_{\text {up }}-M_{\text {up }}$, or $M_{\text {down }}-M_{\text {down }}$, can be effectively read. In fact, the two correspondent $R$ minima are well separated by a relative maximum, the amplitude of which, in all cases, exceeds $140 \mathrm{k} \Omega$ ( $\Delta_{\text {up }}$ and $\Delta_{\text {down }}$ in Figure 6).

The running $M_{\mathrm{i}}$ magnets simulate a sequence of registered bits in a magnetic memory and the MPR core works as a reading head, obviously, with a scale factor of about $10^{4}$. Since polymer wires with diameter of 100-200 nm have been already produced [22], as well as iron nanoparticles assembly could maintain magnetic stability down to size of $10-20 \mathrm{~nm}$ [23], even if its realization is complex, in 
principle it is not to be excluded that the miniaturization of the MPR sensitive core, described in this paper, can achieve similar functionality.

\section{Conclusions}

A MPR magneto-elastomer constituted by Fe microparticles dispersed into a silicone matrix, at the volume percentage near percolation threshold, was produced on the basis of previous investigation, selecting the particle shape in order to have two main effects: i) improve the capability of entrapping a thin silicone layer among the particles in spite of the high particle density; ii) to give a good magnetization intensity at a moderate external field with a practically reversible magnetization process. A proper experimental system was built in which the MPR material is used as core of a sensor. The last is able to read the magnetization direction in a random sequence of little permanent magnets, which are running orthogonally to the sample longitudinal axis. The sensitive core has a constant square section and its length was properly dimensioned in order that a half of the MPR sample is statically magnetized by a fixed permanent magnet, while the opposite half sample is influenced by the magnetizing field of the external magnets whose magnetization direction should be detected. In this way, the interaction between the two sample halves produces a different elastomagnetic strain distinguishing the external magnetization direction concordant or opposite to the permanent one. In turn, these strains produce different resistance changes which can be detected to determine the exact sequence of polarization directions. So that, if the little magnets are arranged in a binary code, it is easy to read any registered sequence. Taking into account the demonstrated sensitivity (about $1250 \mathrm{k} \Omega / \mathrm{T}$ ), the obtained results constitute a stimulating basis to study the effects of reduction in size, going down to nano-range, on the reported principles and experimental methodology, deeply investigating the potentiality of MPR nanowire (also of different composition with respect that reported in this study) in perspective of innovative and cheap reading heads.

\section{References}

[1] Song P., Peng Z-J., Yue Y-L., Zhang H., Zhang Z., Fan Y-C.: Mechanical properties of silicone composites reinforced with micron- and nano-sized magnetic particles. Express Polymer Letters, 7, 546-553 (2013).

DOI: $10.3144 /$ expresspolymlett.2013.51
[2] Bokobza L.: Multiwall carbon nanotube-filled natural rubber: Electrical and mechanical properties. Express Polymer Letters, 6, 213-223 (2012).

DOI: $10.3144 /$ expresspolymlett.2012.24

[3] Stassi S., Canavese G.: Spiky nanostructured metal particles as filler of polymeric composites showing tunable electrical conductivity. Journal of Polymer Science Part B: Polymer Physics, 50, 984-992 (2012). DOI: $10.1002 /$ polb.23091

[4] Wang J., Dai J., Yarlagadda T.: Carbon nanotube-conducting-polymer composite nanowires. Langmuir, 21, 9-12 (2005).

DOI: $10.1021 / 1 \mathrm{a} 0475977$

[5] Wang L., Li Y.: A review for conductive polymer piezoresistive composites and a development of a compliant pressure transducer. IEEE Transactions on Instrumentation and Measurement, 62, 495-502 (2013).

DOI: 10.1109/TIM.2012.2215160

[6] Wang L., Li J.: A piezoresistive flounder element based on conductive polymer composite. Sensors and Actuators A: Physical, 216, 214-222 (2014).

DOI: 10.1016/j.sna.2014.05.010

[7] Patil S. J., Adhikari A., Baghini M. S., Rao V. R.: An ultra-sensitive piezoresistive polymer nano-composite microcantilever platform for humidity and soil moisture detection. Sensors and Actuators B: Chemical, 203, 165-173 (2014).

DOI: $10.1016 /$ j.snb.2014.06.110

[8] Abyaneh M. K., Ekar S., Kulkarni S. K.: Piezoresistivity and mechanical behavior of metal-polymer composites under uniaxial pressure. Journal of Materials Science Research, 1, 50-58 (2012).

DOI: $10.5539 /$ jmsr.v1n3p50

[9] Ausanio G., Barone A. C., Campana C., Iannotti V., Luponio C., Pepe G. P., Lanotte L.: Giant resistivity change induced by strain in a composite of conducting particles in an elastomer matrix. Sensors and Actuators A: Physical, 127, 56-62 (2006). DOI: $10.1016 /$ j.sna.2005.12.002

[10] Gong S., Zhu Z. H.: Giant piezoresistivity in aligned carbon nanotube nanocomposite: Account for nanotube structural distortion at crossed tunnel junctions. Nanoscale, 7, 1339-1348 (2015). DOI: $10.1039 / \mathrm{C} 4 \mathrm{NR} 05656 \mathrm{~F}$

[11] Boundenne A., Mamunya Y., Levchenco V., Garnier B., Lebedev E.: Improvement of thermal and electrical properties of Silicone-Ni composites using magnetic field. European Polymer Journal, 63, 11-19 (2015). DOI: $10.1016 /$ j.eurpolymj.2014.11.032

[12] Gaska K., Kmita G., Rybak A., Sekula R., Goc K., Kapusta C.: Magnetic-aligned, magnetite-filled epoxy composites with enhanced thermal conductivity. Journal of Materials Science, 50, 2510-2516 (2015).

DOI: $10.1007 / \mathrm{s} 10853-014-8809-8$ 
[13] Ruiz M., Marchi C., Perez O., Jorge G., Fascio M., D’Accorso N., Negri M.: Structured elastomeric submillimeter films displaying magneto and piezo resistivity. Journal of Polymer Science Part B: Polymer Physics, 53, 574-586 (2015).

DOI: $10.1002 /$ polb.23672

[14] Knaapila M., Høyer H., Kjelstrup-Hansen J., Helgesen G.: Transparency enhancement for photoinitiated polymerization (UV Curing) through magnetic field alignment in a piezoresistive metal/polymer composite. ACS Applied Materials and Interfaces, 6, 3469-3476 (2014).

DOI: $10.1021 / \mathrm{am} 405625 \mathrm{z}$

[15] Lanotte L., Ausanio G., Iannotti V., Pepe G., Carotenuto G., Netti P., Nicolais L.: Magnetic and magnetoelastic effects in a composite material of Ni microparticles in a silicone matrix. Physical Review B, 63, 054438/1-054438/6 (2001).

DOI: 10.1103/PhysRevB.63.054438

[16] Lanotte L., Ausanio G., Iannotti V., Luponio C.: Influence of particle pre-orientation on elastomagnetic effect in a composite material of ellipsoidal Ni microparticles in a silicone matrix. Applied Physics A, 77, 953-958 (2003).

DOI: $10.1007 / \mathrm{s} 00339-002-1939-\mathrm{x}$

[17] Lanotte L., Ausanio G., Hison C., Iannotti V., Luponio C.: The potentiality of composite elastic magnets as novel materials for sensors and actuators. Sensors and Actuators A: Physical, 106, 56-60 (2003). DOI: 10.1016/S0924-4247(03)00133-X
[18] Filipcsei G., Csetneki I., Szilágyi A., Zrínyi M.: Magnetic field-responsive smart polymer composites. Advances in Polymer Science, 206, 137-189 (2007). DOI: $10.1007 / 122006104$

[19] Zrinyi M.: Magnetically responsive polymer gels and elastomers: Properties, synthesis and applications. in 'Smart polymers and their applications' (eds: de Aguilar M. R., Roman J. S.) Woodhead Publishing, Cambridge, Part 1, 134-165 (2014) DOI: $10.1533 / 9780857097026.1 .134$

[20] Ausanio G., Hison C. L., Iannotti V., Lanotte L., Lanotte L.: Magneto-piezoresistance in elastomagnetic composites. Journal of Applied Physics, 110, 063903/1063903/5 (2011).

DOI: $10.1063 / 1.3634120$

[21] Ausanio G., Iannotti V., Ricciardi E., Lanotte L., Lanotte L.: Magneto-piezoresistance in magnetorheological elastomers for magnetic induction gradient or position sensors. Sensors and Actuators A: Physical, 205, 235-239 (2014).

DOI: $10.1016 /$ j.sna.2013.10.009

[22] Liu B-T., Huang S-X., Lai M-F., Wei Z-H.: Synthesis of $\mathrm{Ag}-\mathrm{Ni}$ core-shell nanowires and their application in anisotropic transparent conductive films. RSC Advances, 5, 1684-1689 (2015)

DOI: $10.1039 /$ C4RA11866A

[23] Allia P., Barrera G., Tiberto P., Nardi T., Leterrier Y., Sangermano M.: $\mathrm{Fe}_{3} \mathrm{O}_{4}$ nanoparticles and nanocomposites with potential application in biomedicine and in communication technologies: Nanoparticle aggregation, interaction, and effective magnetic anisotropy. Journal of Applied Physics, 116, 113903/1-113903/9 (2014).

DOI: $10.1063 / 1.4895837$ 